\title{
Erratum to: Role of dopamine projections from ventral tegmental area to nucleus accumbens and medial prefrontal cortex in reinforcement behaviors assessed using optogenetic manipulation
}

\author{
Xiao Han ${ }^{1} \cdot$ Man-yi Jing ${ }^{1} \cdot$ Tai-yun Zhao ${ }^{1} \cdot \operatorname{Ning~} \mathrm{Wu}^{1} \cdot \operatorname{Rui} \operatorname{Song}^{1} \cdot{\operatorname{Jin~} \mathrm{Li}^{1}}^{1}$
}

Published online: 7 August 2017

(C) Springer Science+Business Media, LLC 2017

Erratum to: Metab Brain Dis (2017)

https://dx.doi.org/10.1007/s11011-017-0023-3

The authors woud like to indicate the below corrections to the above referenced article.

In the second paragraph of section "Activation of the dopaminergic projection from VTA to NAc core and shell", $P<0.0001$ should changed to $P<0.001$. The corrected first sentence should read:

Time main effect: $F_{29,232}=6.82, P<0.001$; Interaction (Pokes $\times$ Time): $F_{29,232}=6.77, P<0.001$.

In the section "Micro-injection of METH into the NAc (core and shell) and mPFC (IL and PL) induced hyperactivity", $F_{3,23}=1.858$ should be changed to $F_{3,20}=1.858$. The corrected first sentence should read:

Micro-injections of METH $(2-8 \mu \mathrm{g})$ in the NAc core and NAc shell significantly enhanced locomotor activity in a dose dependent manner (Fig. $4 \mathrm{a}, \mathrm{b} ; F_{3,32}=6.444$, $P=0.002$, and $n=9$ in each group and $F_{3,36}=6.66$, $P=0.001$, and $n=10$ in each group, respectively, as assessed by one-way ANOVA) but not for $\operatorname{mPFC}\left(F_{3,20}=1.858\right.$, $P>0.05$, and $n=6$ in each group and $F_{3,28}=0.715$, $P>0.05$, and $n=8$ in each group, respectively, as assessed by one-way ANOVA).

Also, Figs. 2e, f and 3e, f are incorrect. The corrected 2 and 3 figures are shown on the following page:

The online version of the original article can be found at https://dx.doi. org/10.1007/s11011-017-0023-3

Rui Song

songrui1983@yeah.net

$\checkmark$ Jin Li

jinli9802@163.com

State Key Laboratory of Toxicology and Medical Countermeasures, Beijing Key Laboratory of Neuropsychopharmacology, Beijing Institute of Pharmacology and Toxicology, 27th Taiping Road,

Beijing 100850, China 
A

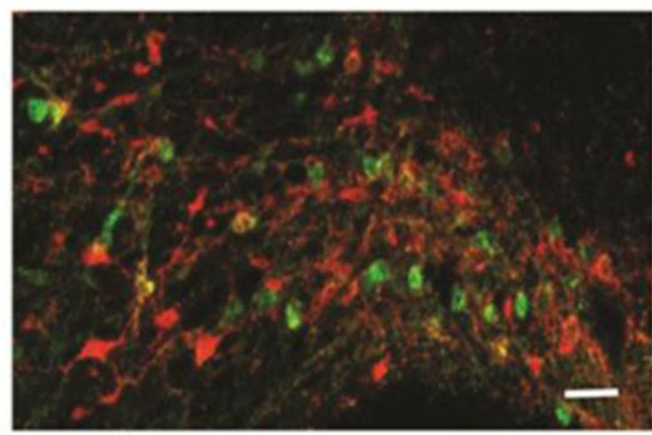

C

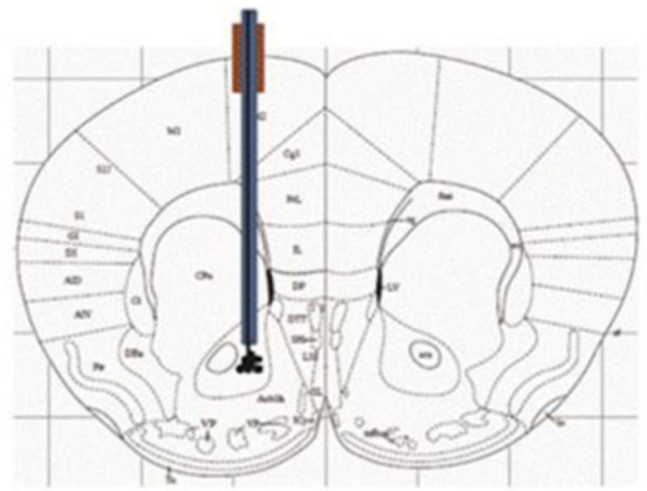

E

(NAc core)

- ChR2/mCherry-active pokes $(n=9)$

- - ChR2/mCherry-nactive pokes $(n=9)$

$\checkmark$ mCherry-active pokes $(n=5)$

$\triangle$ m Cherry-inactive pokes $(n=5)$

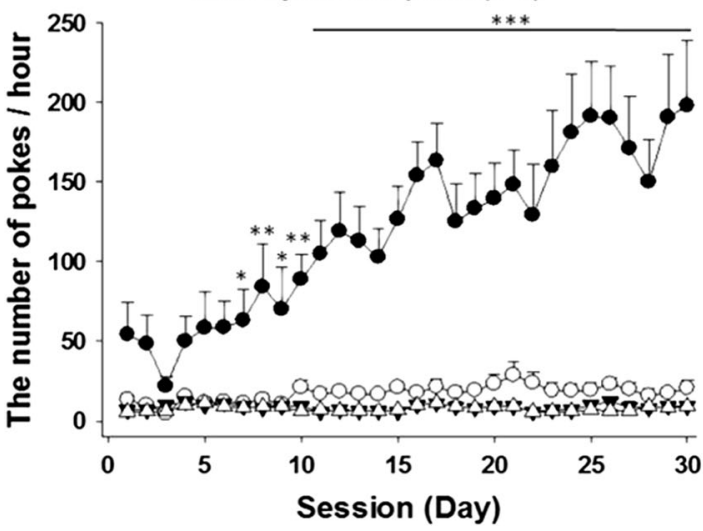

Fig. 2 Optogenetic self-stimulation of the DA projection from the VTA into the NAc (core and shell). a Coronal image co-localization of ChR2mCherry with TH expressing neurons in the VTA. Scale bar $50 \mu \mathrm{m}$. b Examples show a distribution of ChR2-mCherry-expressing axons in the NAc. Scale bar $500 \mu \mathrm{m}$. c and $\mathbf{d}$ Schematic of optic fiber placement above the NAc core and NAc shell. $\mathbf{e}$ and $\mathbf{f}$ Activation of the projection from the
B (NAc)

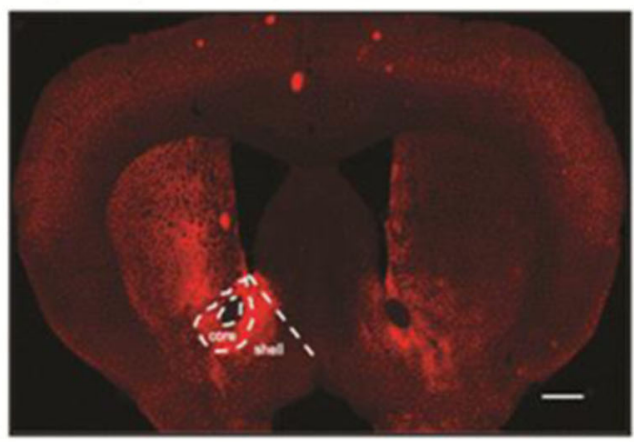

D (NAc shell)

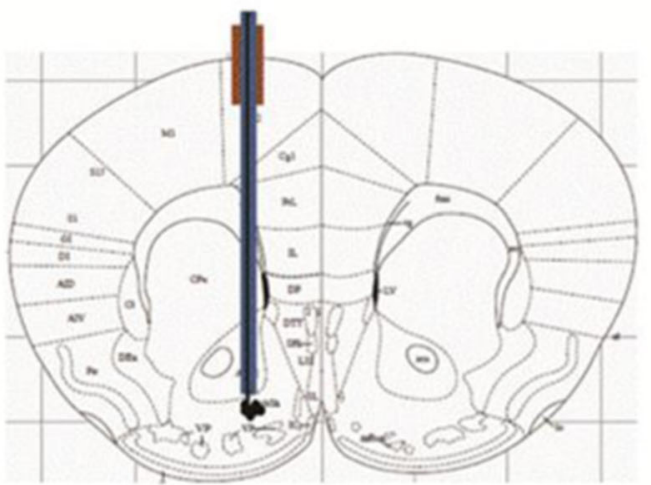

$\mathbf{F}$

(NAc shell)

- ChR2/mCherry-active pokes ( $n=7)$

-0 ChR2/mCherrytnactive pokes $(n=7)$

$\rightarrow-$ mCherry-active pokes $(n=5)$

$\triangle \backsim$ mCherry-inactive pokes $(n=5)$

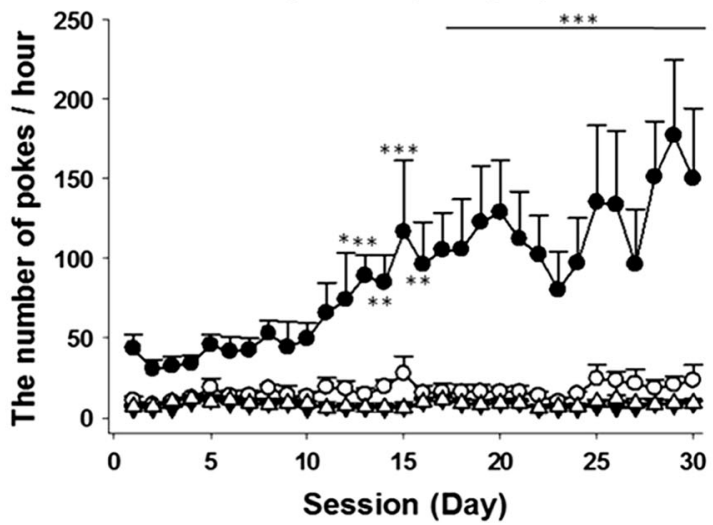

VTA into the NAc core and shell significantly established stable selfstimulation behavior (laser stimulation $473 \mathrm{~nm}, 60$ pulses of $15 \mathrm{~ms}$, at $20 \mathrm{~Hz}$ ) during $60 \mathrm{~min}$ sessions for 30 days. ${ }^{*} P<0.05,{ }^{* * *} P<0.001$, compared with inactive pokes as assessed by two-way ANOVA followed by Bonferroni's test 
A (VTA)

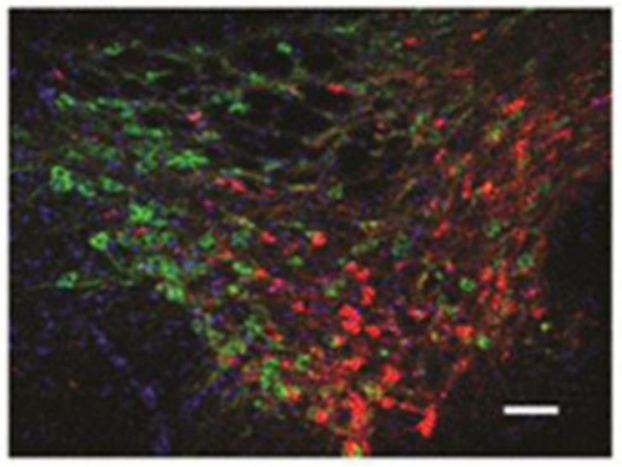

C

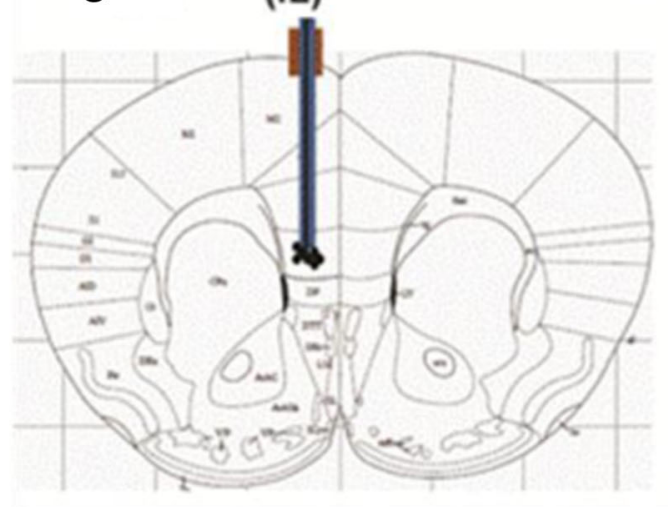

E

(IL)
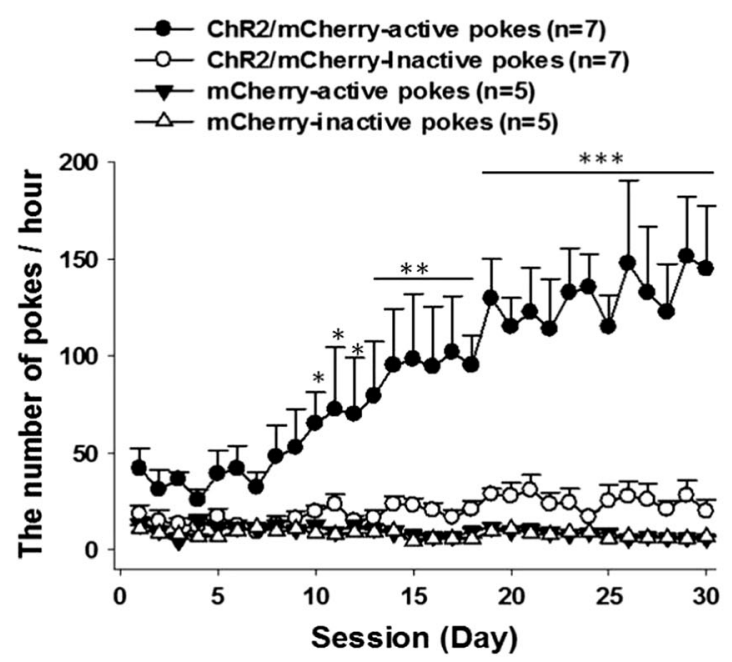

Fig. 3 Optogenetic self-stimulation of the DA projection from the VTA into the mPFC (IL and PL). a Coronal image co-localization of ChR2mCherry with TH expressing neurons in VTA. Scale bar $50 \mu \mathrm{m}$. b Examples show a distribution of ChR2-mCherry-expressing axons in the mPFC. Scale bar $200 \mu \mathrm{m}$. c and d Schematic of optic fiber placement above the IL and PL. e and $\mathbf{f}$ Activation of the projection from the VTA
B $\quad(m P F C)$

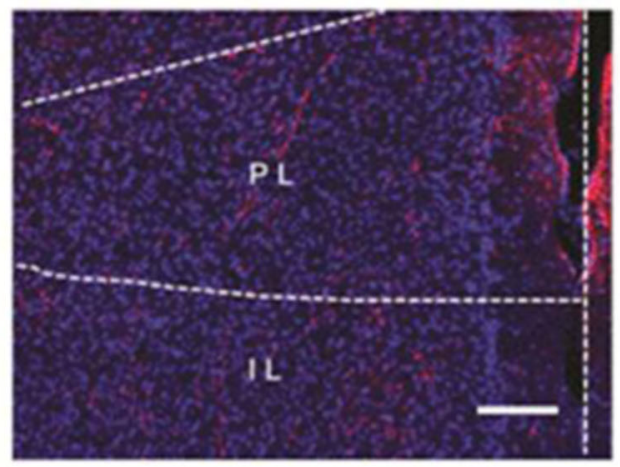

D

(PL)

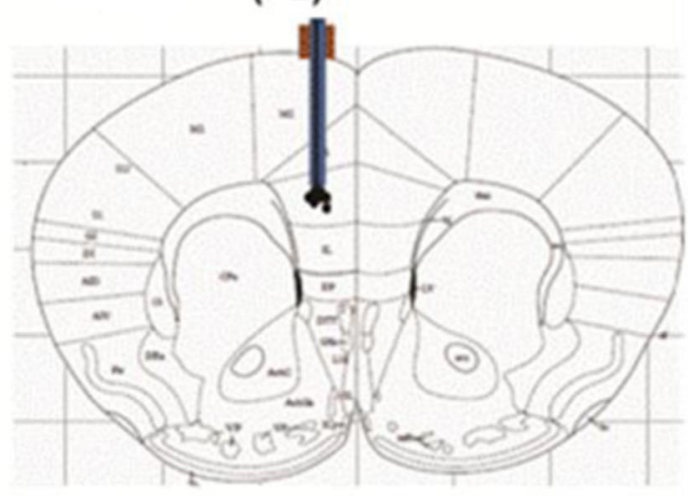

$\mathbf{F}$

(PL)

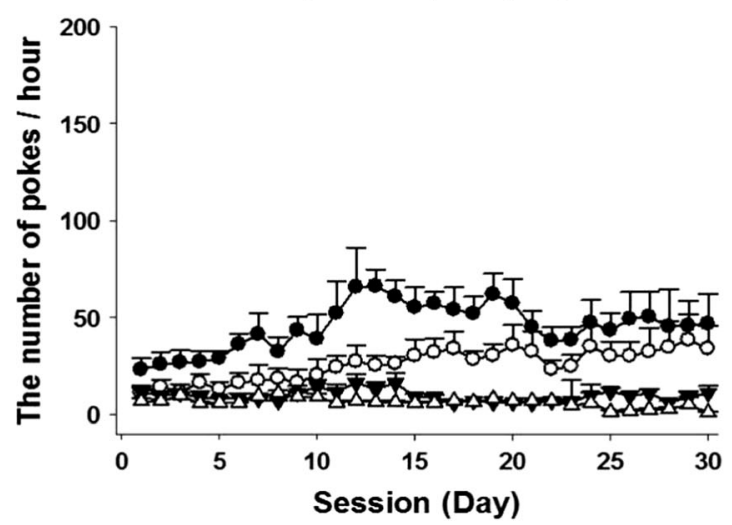

into the IL (but not into the PL with the same conditions), significantly established stable self-stimulation behavior (laser stimulation $473 \mathrm{~nm}, 60$ pulses of $15 \mathrm{~ms}$, at $20 \mathrm{~Hz}$ ) during $60 \mathrm{~min}$ sessions for 30 days. ${ }^{*} P<0.05$, ${ }^{* * *} P<0.001$, compared with inactive pokes as assessed by two-way ANOVA followed by Bonferroni's test 